\title{
A qualitative study of the experience of oral cancer among Taiwanese men.
}

Running title: oral cancer experiences in Taiwan

\section{Tsun-Wen Hu, RN MN}

School of Nursing and Midwifery, Griffith University, Nathan, Queensland, Australia

\section{Marie Cooke, RN PhD}

Research Centre for Clinical and Community Practice Innovation, Griffith University,

Queensland, Australia

\section{Alexandra McCarthy, RN PhD}

School of Nursing, Queensland University of Technology, Queensland, Australia

Corresponding author:

Tsun-Wen Hu,

School of Nursing and Midwifery,

Griffith University,

Nathan Campus,

170 Kessels Road,

Nathan, QLD 4111,

Australia.

Email: redwing2004@hotmail.com 


\section{A qualitative study of the experience of oral cancer among Taiwanese men.}

\section{ABSTRACT}

The incidence and mortality of oral cancer in Taiwanese men has increased over the past decade, primarily associated with a surge in the popularity of betel quid chewing. The aim of this study was to examine the experience of six Taiwanese men with oral cancer, who were aged between 40 and 60 years, using a qualitative approach. The three major themes emerging from the data include: (i) understanding the cancer diagnosis; (ii) the challenges of cancer treatment; and (iii) adapting to difference. Increasing nurses' understanding of the experiential aspects of oral cancer in this population is required if nurses are to develop successful health promotion programmes and nursing interventions to meet these patients’ needs. (130 words)

Key words: adaptation, health behaviour; mouth neoplasms; piper betle; Taiwan. 


\section{INTRODUCTION}

Oral cancer affects $~ 350,000-400,000$ people worldwide annually, and the incidence is increasing. ${ }^{(1)}$ It is the sixth most common cancer and a major cause of cancer morbidity and mortality worldwide, ${ }^{(2)}$ representing about $5.5 \%$ of malignancies. ${ }^{(3)}$ Taiwan has recorded a marked increase in the incidence and mortality of oral cancer over the past decade. ${ }^{(4)} \mathrm{A}$ significant risk factor for the development of oral cancer is betel quid chewing, a widespread habit throughout Southeast Asia. Taiwan has a high proportion of betel quid chewers, and $80 \%$ of oral cancer deaths in Taiwan are associated with this habit. ${ }^{(5,6)}$

Oral cancer is defined as any malignant neoplasm of the oral cavity. ${ }^{(1)}$ Genetic susceptibility and external agents, including smoking, alcohol, areca nut/betel quid chewing, dietary factors and viruses may all play a role in its development. ${ }^{(7-9)}$ Betel quid chewing is also a major risk factor for precancerous oral lesions, such as oral leukoplakia and oral submucous fibrosis. ${ }^{(10,11)}$ Precancerous oral lesions appear to be exacerbated by the synergy of smoking and betel quid chewing ${ }^{(5)}$. Whilst it has been implicated, hard evidence is currently lacking regarding the correlation of the development of precancerous lesions with alcohol intake. ${ }^{(11)}$ 
Betel quid composition varies, but mostly consists of arecoline, guvacoline, lime, and Piper betle flower. Its principle action is upon the central and autonomic nervous systems. Chewing quid produces a sense of well-being, euphoria, heightened alertness, sweating, salivation, increased body temperature, reduced hunger and fatigue, and increased capacity to work. It is widely believed to assist in adaptation to emotional or stressful situations. It also causes habituation, addiction, and withdrawal symptoms, such as low spirits, general body discomfort, loose teeth, loss of concentration and bad temper. ${ }^{(12)}$

Betel quid chewing was traditionally associated with lower education and income levels in the Taiwanese context. ${ }^{(13,14)}$ The habit has recently extended to younger generations and to people with higher education, and is increasingly widespread in all areas of Taiwan. ${ }^{(15)}$ Betel quid chewing usually starts in adolescence, increases after high school, and peaks between the ages of 30 and 49 years. ${ }^{(13)}$ Most students start chewing betel quid due to factors such as curiosity, peer influence, keeping warm and emulating adult behaviour; unfortunately, many are ignorant of the harmful health effects. ${ }^{(15)}$ More males than females are betel quid chewers, ${ }^{(15),(16)}$ a habit that is frequently accompanied by cigarette smoking and drinking alcohol. ${ }^{(17)}$ Notably, all 18-year-old males in Taiwan undertake a two-year military term, where the pressure to engage in habits of smoking, drinking and chewing betel quid is common. 
In 2001, nearly one in four adult males in Taiwan aged between 25 and 64 years chewed betel quid. Nearly all betel quid chewers also smoked cigarettes, but only one third of smokers chewed quid. ${ }^{(13)}$ Most chewers begin smoking before chewing, thus smoking appears to be a precursor for betel quid chewing. On average, those who chew betel quid also smoke more cigarettes per day than non-chewers. ${ }^{(13)}$ As betel quid in Taiwan contains no nicotine, chewing is not an alternative to smoking, but an adjuvant. ${ }^{(18)}$ Evidence suggests that male chewers think that betel chewing projects a strongly masculine image. ${ }^{(19,20)}$ In some parts of Taiwan, cigarettes and betel quid are offered as a greeting for initiating and promoting interpersonal relationships. It would also appear that the stresses of daily life become tolerable with the relief of 'a chew'. (20)

Oral cancer in Taiwan occurs in a 6:1 male to female ratio. ${ }^{(14,21)}$ In a recent study of 1010 patients, the average age of diagnosis was 51.7 years, with peak age of occurrence being 50 to 59 years, followed by the group aged 40-49 years. ${ }^{(14)}$ Many Taiwanese initially present for medical care with an advanced stage of oral cancer; the most likely reason for the delay in seeking medical treatment is that patients often first try traditional Chinese medicine. ${ }^{(21)}$ 
Oral cancer poses enormous challenges to body image and self-esteem through the physically disfiguring and disabling effects of both the disease and its treatment. Permanent facial alterations due to surgery and radiotherapy can impair chewing, swallowing and speaking, as well as sight, smell and hearing. Adjuvant chemotherapy and radiotherapy are widely used to prevent the spread of malignancy and to prevent the progression of premalignant lesions; side effects may include extensive ulceration of the oral mucosa and alteration of facial structures, resulting in temporary or permanent difficulties in talking, swallowing and maintaining oral hygiene. ${ }^{(7,22)}$

In summary, the rising incidence of oral cancer among middle-aged Taiwanese males is a major healthcare issue. Few investigations have explored the subjective experience of oral cancer and coping with the resultant facial disfigurement for these men. We conducted this research to examine the experience of oral cancer for men in Taiwan.

\section{METHOD}

This study was conducted through the Ear, Nose and Throat (ENT) outpatient clinic of a teaching hospital in mid-Taiwan. Ethical approval was obtained from the university and hospital ethics committees. 
The aim of the study was to provide in-depth depictions of the experience of oral cancer of Taiwanese men, an area about which currently little is known. As such, a descriptive, qualitative, thematic analysis approach was used to provide a beginning understanding of these issues. Inclusion criteria included Taiwanese males aged 40-60 years with stage I - III non-terminal oral cancer. Those who met criteria were invited to participate in the study through the provision of an information sheet by the ENT doctor, who was not connected with the research. Those who agreed to be interviewed returned a slip with contact details to the doctor and were subsequently contacted by the researcher. Written consent was obtained prior to interview.

Six participants took part in the study. Data were collected through individual, in-depth, semi-structured interviews, either in a hospital discussion room or the participant’s home. Each participant was interviewed once and each interview took $\sim 1.5$ hours. The interviews were audio-recorded and transcribed verbatim in Chinese. Once categories and themes were generated, these were translated into English by one translator to maximise consistency in translation and credibility in data analysis. ${ }^{(23)}$ Saturation of data was achieved despite the small sample through several hours of interviews to the point where no new information arose. 
Interview material was systematically reviewed to sort and classify data into representational groups that enabled development of categories that focused on behaviour, observation or verbal expression. ${ }^{(24)}$ As relationships among categories, participants, actions and events began to emerge ${ }^{(25)}$ implicit and implied meanings within accounts were analysed into themes. The themes emerged from two or more related patterns of smaller units derived from categories of data. ${ }^{(24)}$

Rigour was achieved through a range of processes. Overall, the research was informed by qualitative research principles that aimed to understand individual perspectives and experiences. ${ }^{(26)}$ Interview content and data analysis were confirmed by way of member checks by participants to ensure credibility. ${ }^{(27)}$ Participants were provided with a summary of the initial phase one analysis of their interview content so that they could read it and confirm the essence of their experience.

\section{RESULTS}

Six men aged from 40 to 57 years took part in this study. Their age at diagnosis ranged from 38 to 51 years. All had experienced stage I to III non-terminal oral cancer and had undergone treatment (surgery with or without radiotherapy) at a hospital in mid-Taiwan. Two participants experienced oral cancer recurrence following the first surgery, and all had som 
degree of facial disfigurement after treatment. All the participants had chewed betel quid, smoked and consumed alcohol for over 20 years at the time of their diagnosis. Five participants were of middle or high socio-economic status with college or lower levels of education; one was of lower socio-economic status, and had received education at junior hight school level.

Three themes emerged from the patterns of categorised interview data including: (i) understanding the cancer diagnosis; (ii) the challenges of cancer treatment; and (iii) adapting to difference.

\section{Theme I: Understanding the cancer diagnosis}

This theme is generated by three patterns of categorised data. These illustrate the participants' experiences of being diagnosed with oral cancer; their realization of how previous risky health behaviours contributed to their diagnosis; and the implications of their unawareness of the early signs and symptoms of oral cancer and subsequent failure to access early treatment. 


\section{Experiencing the diagnosis}

Participants described their initial responses to finding out that they had oral cancer in terms of denial and disbelief such as un-preparedness, shock, apprehension and worry, panic and depression. These feelings related to their fear of the cancer and the impact it had on family members in terms of a sense of hopelessness and potential loss of life aspirations.

I was shocked, worried and depressed then, I thought I was going to die soon and had my will ready for my family... It was hard to actually accept this truth, I took about 3 years to adjust myself and accept all of this

\section{$\underline{\text { Risky health behaviours become known }}$}

All participants recalled a history of smoking, drinking alcohol and chewing betel quid since adolescence. Participants acknowledged that they started to smoke cigarettes, chew betel nut, and drink alcohol at around 18 years of age while serving their mandatory 2 year military term. They considered these normal activities that allowed them to make friends and maintain social relationships. They described how continuing to chew betel quid after army discharge helped them to cope with the pressures of needing to maintain their livelihoods and work performance at high levels. Participants’ comments provided us with insights into the relationship between risky habits, irregular life patterns, and the demands of social relationships and work. At the time of their diagnosis, however, the participants appeared to 
have little understanding of the links between their health behaviours and oral cancer. That understanding only developed once they had experienced cancer treatment.

I started smoking and drinking while serving in the army, partly being young and partly out of curiosity. I have to take night shifts at work, and chewing betel quid makes me refreshed and energized.

I think the reason I got oral cancer is because I chew betel quid, love heavily flavoured food and never get enough sleep out of extended night shifts.

\section{$\underline{\text { Lack of knowledge of oral cancer }}$}

On reflection, all participants recalled their awareness of seemingly harmless oral lesions before they were diagnosed. None believed these were signs of cancer or believed they warranted medical attention, hence most initially sought Chinese medicine or over-the-counter ointment. One participant initially visited the dentist to have his oral lesion investigated, but was not referred for medical assessment for several months.

Any information about oral cancer was not quite well known yet when I got oral cancer. I only knew it was a cancer, but nothing about how serious it was. The number of oral cancer patients was not as many as today. 
I found a white patch at the mouth floor and it was painful. I went to a pharmacy to get oral ointment to apply on it. I also applied Chinese herb on it, however, few months later, the pain was not relieved and getting worse.

\section{Theme II: The challenges of cancer treatment}

All participants had their lesions surgically resected, with a further two participants undergoing adjuvant radiotherapy. Both treatment modalities can result in significant facial and functional deficits, and these were clearly described by the participants in terms of the consequent challenges they faced in everyday activities such as speaking, eating and working during and immediately after their treatment. They also articulated the challenges experienced in relationships and self-esteem from their altered speech and appearance.

\section{Challenges in everyday activities}

Participants' dietary habits were universally affected as a result of the treatment and its side effects. All participants could only consume liquid or soft diets during treatment, and after it was completed. The everyday eating activity that they had previously taken for granted and enjoyed had now become a source of frustration and resentment. In addition, four of the men experienced restricted mouth movement post-surgery, resulting in speech impediments. Their inability to make themselves understood in daily communication with friends, relatives 
and business colleagues, both in person and on the telephone, compounded their feelings of frustration and anger. In many instances their cancer treatment had altered their appearance. As a result, their income and employment opportunities were profoundly affected not just in relation to their difficulties with speech but due to their withdrawal from an active role in their businesses due to others’ apparently negative attitudes towards their appearance.

I did not used to eat such things as congee ${ }^{1}$ or any soup before.

I hated to eat such food, but I eat congee and soup now ... I know it is impossible to eat the same food as I liked before.

Because of surgery, I have difficulties in pronunciation ... At first my wife could not understand my meaning at all, I even got mad at her.

When I was looking for a new job, I encountered great difficulties, for instance, while I was interviewed face to face ... It is not easy to pass an interview; being treated with discrimination, being rejected, and sarcasm and spite are inevitable. In fact, people still hold a discriminatory attitude towards the population with facial disfigurement in our society; so disfigured people usually meet the toughest challenge in terms of seeking a job.

\section{Challenges in relationships and self-esteem}

This disfigurement caused by oral cancer and its treatment posed a particularly $-12$

${ }^{1}$ Congee: a Taiwanese version of rice porridge. 
significant challenge to the participants' sense of self, as they often experienced feelings of diminished self-esteem and self-confidence. The data showed how appearance, feelings of difference and ability to speak clearly influenced their self-esteem and self-confidence, with many becoming socially isolated and deliberately withdrawing from relationships.

... my face seems seriously asymmetric and scars are visible. This is what I was most concerned about and it was the most difficult part for me to adjust to. I think it indeed greatly impacts on a man who is in his forties like me, because men in their forties tend to have more vulnerable self-esteem than at other stages ... I do not want to go out.

I felt very depressed and angry with my altered appearance, because I was totally unprepared; it was painful for me to accept this. The reason I felt depressed was that I saw myself slobbering...everyday... and that has affected my self-esteem.

\section{Theme III: Adapting to difference}

Participants used a variety of strategies to adjust to their new appearance and the reality of living with cancer. These included trying to maintain a positive attitude; developing self-reliance; accepting their appearance; modifying their lifestyle; setting new life goals; and seeking knowledge about healthy living. Three have become cancer volunteers at the hospital where they were treated. 
...my health is more important now, I have already quit all bad habits, I eat much healthier and live a regular life pattern...

I am going to carry out my plans after the plastic surgery has made my face not so obviously different, such as to get a new car and a job again.

...I am only 40. So I tell myself just face the music...I never think it is a burden; it is a part of my life now.

...I believe that being optimistic towards the illness is the most important thing and to not expect someone else to do things for you.

The most significant adaptive resource the participants described was the support obtained from their families and friends, fellow patients and health care providers, as well as from their religious beliefs. They generally agreed that family was the most helpful source of emotional support. Although the participants appreciated health professionals' support, expertise, attitudes, and explanations they suggested ways health professionals could improve the system for future oral cancer patients. These included: clear identification of health providers' responsibilities, improvement in communication skills, and better development of therapeutic relationships with patients. 


\section{DISCUSSION}

These findings provide valuable insights in relation to the development of health education programs and nursing interventions for those at risk of, or diagnosed with, oral cancer in Taiwan. Most participants came from middle to high Taiwanese social strata, reflecting other research that indicates that the betel chewing habit is gradually spreading to those with higher education in Taiwan. ${ }^{(15)}$ Hence this group should now be considered as being at high risk for developing oral cancer when considering public and school health programs.

Studies have found that patients may delay seeking medical treatment for oral cancer in Taiwan, and may ignore small and less painful oral lesions or initially seek traditional Chinese medicine or over-the-counter ointments ${ }^{(21)}$; our findings support this. Most of the men were unaware of the signs of oral cancer and associated risk factors prior to diagnosis. While participants learned after diagnosis that risky habits probably caused their oral cancer, and all discontinued their betel habit as a result, some continued to consume alcohol and tobacco and did not appear to comprehend the synergistic role of these substances in the development of oral cancers. This finding is corroborated by international data. An investigation into the health behaviours of 264 head and neck patients in the Netherlands found that $50 \%$ of patients continued to smoke and $80 \%$ continued to drink alcohol following diagnosis. The authors 
attributed this to the anxiety and depression associated with diagnosis and a lack of understanding regarding risk management. ${ }^{(28)}$ Given the high levels of anxiety and depression displayed by many cancer patients after treatment, interventions designed to reduce risky behaviour should therefore address the accompanying psychological stress of diagnosis.

Functional deficits caused by initial alterations to facial structures greatly affected the participants' ability to eat during the post-operative period, threatening their nutritional status and subsequent healing and recovery. In addition, radiotherapy results in immediate alterations to salivary function significantly affecting chewing and swallowing, and which may be temporary or permanent. This highlights a need for early and ongoing nutritional assessment and intervention. We would recommend that nurses integrate nutritional assessment from admission, and continue to assess this during treatment, and after active treatment has ceased. ${ }^{(29)}$ Educating patients on mouth opening exercises, meticulous dental care, salivary substitution and demonstrating ways to adapt eating could also be beneficial.

Four participants reported impaired speech, severely affecting their communication and psychosocial functioning. This reflects international studies reporting that communication difficulties are a major source of psychological distress for patients with head and neck cancer. ${ }^{(30)}$ In this context, none of the participants received prophylactic speech therapy 
intervention prior to their treatment to help prepare them for this eventuality; nor were they offered post-treatment speech therapy services. We suggest that speech therapy and education should be integrated into routine health and nursing care for this population.

All participants described challenges maintaining income and employment. Some owned businesses and were wholly responsible for the maintenance of the family income during their illness, which they reported as an enormous burden. Others encountered issues when they attempted to seek employment, such as pointed questions about their capacity to work, and discriminatory attitudes towards illness and disfigurement. Although the National Health Insurance of Taiwan covers most treatment fees for people with severe illnesses, the reduction in income could be considerable, especially for those with low socio-economic status. In Taiwan, males usually provide the main source of household income, and so their diagnosis and treatment affects the family's financial situation. While this is critical for low-income workers, we found the middle to high income-earners also experienced this impact.

Participants reported diminished self-esteem and self-confidence, as well as anger, frustration and depression. They claimed to feel more vulnerable in terms of self-esteem in the face of others' reactions than they might have at other stages of their lives. In line with this finding, Davidson and McCabe assert that men in their 30s and 40s are considered more 
vulnerable to dissatisfaction with their changed bodies. ${ }^{(31)}$ The perceived importance of appearance in social relationships is a major cause of deep psychological distress for many oral cancer patients. ${ }^{(32)}$ The reactions of others, who often recoil and feel uncomfortable when confronted with visible difference, have a profound effect. ${ }^{(33)}$

Although participants tried to employ positive coping strategies, depression and withdrawal were common. The ability to cope with stressors is influenced by the resources available, including health and energy; levels of confidence; and social support. ${ }^{(34)}$ Participants generally agreed that family was the most essential source of support. In this way, nursing care should be planned with the whole family as they can also be affected by a patient's illness and adversely influence their recovery. ${ }^{(35)}$ Fellow cancer patients also provided valuable support by sharing experiences and exchanging self-care information and knowledge of resources.

Unfortunately, participants reported inadequate psychological and educational support from healthcare providers during hospitalisation and after discharge. Whether this is due to healthcare professionals failing to understand the importance of providing such support, ${ }^{(36)}$ or the lack of appropriate psychosocial skills training, ${ }^{(37)}$ is unclear and warrants further 
research.

\section{CONCLUSION}

This study provides an insight into Taiwanese men's experiences of oral cancer, although some limitations are recognised in the findings. The participants were recruited from one setting, which could limit the transferability of the findings. Although these findings have emerged from the Taiwanese context, they might be applicable to other countries where betel chewing is widespread.

Early education instituted in the military and high schools focussing on the risks of smoking and betel quid chewing, and the identification and treatment of symptoms may help reduce the prevalence of oral cancer. Taiwanese health practitioners such as dentists may also benefit from education about the importance of early diagnosis and referral, particularly when there is a history of risk factors. It is also clearly important that health promotion programs in this context should recognise and incorporate the preference of many Taiwanese people for the philosophies and practices of traditional Chinese medicine. We would argue that Chinese and Western philosophies are not necessarily incompatible, but that there is a challenge inherent in melding the two to ensure best preventative outcomes in this high risk group of men. 
The psychosocial and economic impact of oral cancer is enormous for patients and families. Nurses must appreciate the support derived from families and friends, fellow patients, health care providers and religious beliefs, and incorporate these into care where possible. Healthcare professionals need training in communication skills and providing psychological support. ${ }^{(36)}$ Finally, discharged patients should be referred to community nurses, who play a unique role in assessing and assisting recovery, and can make further referrals if needed.

\section{ACKNOWLEDGEMENTS}

The authors would like to thank the Otorhinolaryngology-Head and Neck surgeon Dr.

Yung-Sung Wen (Attending physician, Department of Otorhinolaryngology-Head and Neck Surgery; Director of Faculty Development, Department of Medical Education \& Research) who helped the research team recruit participants and obtain the approval from the IRB of the hospital in Taiwan. 


\section{REFERENCES}

1. Subramaniam S, Smith K, Kruger E, Tennant M. Hospitalization for oral malignancies in Western Australians: A four-year retrospective analysis. Asia-Pacific Journal of Clinical Oncology 2005;1:151-7.

2. Nair S, Pillai M. Human papillomavirus and disease mechanisms: Relevance to oral and cervical cancers. Oral Disease 2005;11:350-9.

3. Speight P, Farthing P, Bouquot J. The pathology of oral cancer and precancer. Current Diagnostic Pathology 1996;3:165-76.

4. Taiwan Department of Health. Statistics Causes of Death. 2006;1. Available at: http://www.doh.gov.tw/statistic/index.htm. Accessed on 18 March 2007.

5. Chang Y, Hu C, Tseng T, Tai K, Lii C, Chou M. Synergistic effects of nicotine on arecoline-induced cytotoxicity in human buccal mucosal fibroblasts. Journal of Oral Pathology and Medicine 2001;30:458-64.

6. Ho P, Ko Y, Yang Y, Shieh T, Tsai C. The incidence of oropharyngeal cancer in Taiwan: An endemic betel quid chewing area. Journal of Oral Pathology and Medicine 2002;31:213-9.

7. Das B, Nagpal J. Understanding the biology of oral cancer. Medical Science Monitor 2002;8(11):258-67.

8. Zain R. Cultural and dietary risk factors of oral cancer and precancer: A brief overview. 
Oral Oncology 2001;37:205-10.

9. Chen Y, Huang H, Lin L, Lin C. Primary oral squamous cell carcinoma: An analysis of 703 cases in southern Taiwan. Oral Oncology 1999;35:173-9.

10. Chung C, Yang Y, Wang T, Shieh T, Warnakulasuriya S. Oral precancerous disorders associated with areca quid chewing, smoking, and alcohol drinking in southern Taiwan. Journal of Oral Pathology and Medicine 2005;34:460-6.

11. Lee C, Ko Y, Huang $\mathrm{H}$, et al. The precancer risk of betel quid chewing, tobacco use and alcohol consumption in oral leukoplakia and oral submucous fibrosis in southern Taiwan. British Journal of Cancer 2003;88:366-72.

12. Chu N. Effects of betel chewing on the central and autonomic nervous systems. Journal of Biomedical Science 2001;8:229-36.

13. Wen C, Tsai S, Cheng C, et al. Uncovering the relation between betel quid chewing and cigarette smoking in Taiwan. Tobacco Control 2005b;14(1):116-22.

14. Wong Y, Tsai W, Lin J, et al. Socio-demographic factors in the prognosis of oral cancer patients. Oral Oncology 2006;42(2):893-906.

15. Wang S, Tsai C, Huang S, Hong Y. Betel nut chewing and related factors in adolescent students in Taiwan. Public Health 2003;117:339-45.

16. Lu C, Lan S, Hsieh C, et al. Prevalence and characteristics of areca nut chewers among junior high school students in Changhua county, Taiwan. Community Dentistry and Oral 
Epidemiology 1993;21(6):370-3.

17. Ko Y, Chiang T, Chang S, Hsieh S. Prevalence of betel quid chewing habit in Taiwan and related sociodemographic factors. Journal of Oral Pathology Medicine 1992;21(6):261-4.

18. Wen C, Cheng T, Eriksen M. How opening the cigarette market led to an increase in betel quid use in Taiwan. Public Health 2005;119:940-4.

19. Wang S, Tsai C, Huang S, Hong Y. Betel nut chewing: The prevalence and the intergenerational effect of parental behavior on adolescent students. The Journal of Adolescent Health 2004;34:244-9.

20. Williams S, Malik A, Chowdhury S, Chauhan S. Sociocultural aspects of areca nut use. Addiction Biology 2002;7:147-54.

21. Lo W, Kao S, Chi L, Wong Y, Chang R. Outcomes of oral squamous cell carcinoma in Taiwan after surgical therapy: Factors affecting survival. Journal of Oral and Maxillofacial Surgery 2003;61:751-8.

22. Ord R, Blanchaert R. Current management of oral cancer: A multidisciplinary approach. Journal of the American Dental Association 2001;132:19S-23S.

23. Twinn S. An exploratory study examining the influence of translation in the validity and reliability of qualitative data in nursing research. Journal of Advanced Nursing 1997;26:418-23. 
24. DeSantis L, Ugarriza D. The concept of theme as used in qualitative nursing research. Western Journal of Nursing Research 2000;22(3):351-72.

25. Burns N, Grove S. Understanding Nursing Research. 3rd ed. Philadelphia: Saunders; 2003.

26. Merriam SB. Introduction to qualitative research. In: Merriam SB, ed. Qualitative Research in Practice: Example for Discussion and Analysis. San Francisco: Jossey-Bass; 2002:3-17.

27. Cutcliff J, McKenna H. Establishing the credibility of qualitative research findings: The plot thickens. Journal of Advanced Nursing 1999;30(2):374-80.

28. Tromp D, Brouha X, Hordijk G, et al. Medical care-seeking and health-risk behavior in patients with head and neck cancer: The role of health value, control beliefs and psychological distress. Health Education Research 2005;20(6):665-75.

29. Capra S, Ferguson M, Ried K. Cancer: impact of nutrition intervention outcome—nutrition issues for patients. Nutrition 2001;17(9):769-72.

30. Hutton J, Williams M. An investigation of psychological distress in patients who have been treated for head and neck cancer. The British Journal of Oral and Maxillofacial Surgery 2001;39:333-9.

31. Davison T, McCabe M. Relationships between men's and women's body image and their psychological, social, and sexual functioning. Sex Roles 2005;52(7/8):463-75. 
32. Rumsey N, Clarke A, White P, Wyn-Williams M, Garlick W. Altered body image:

Appearance-related concerns of people with visible disfigurement. Journal of Advanced Nursing 2004;48(5):443-53.

33. Freeman M, Jaoudé P. Justifying surgery’s last taboo: The ethics of face transplants. Journal of Medical Ethics 2007;33:76-81.

34. Lazarus R, Folkman S. Stress, Appraisal, and Coping. New York: Springer; 1984.

35. Shields L, Werder H. Perioperative Nursing. London: Greenwich Medical Media; 2002.

36. Furness PJ. Exploring supportive care needs and experiences of facial surgery patients. British Journal of Nursing 2005;14(12):641-5.

37. Clarke A, Cooper C. Psychosocial rehabilitation after disfiguring injury or disease: Investigating the training needs of specialist nurses. Journal of Advanced Nursing 2001;34(1):18-26. 
